\title{
Effect of pressure on joint cascade of kinetic energy and helicity in compressible helical turbulence
}

\author{
Zheng Yan, ${ }^{1,2}$ Xinliang Li, ${ }^{1,2}$ Jianchun Wang, ${ }^{3}$ and Changping $\mathrm{Yu}^{1,2, *}$ \\ ${ }^{1}$ LHD, Institute of Mechanics, Chinese Academy of Sciences, Beijing 100190, China \\ ${ }^{2}$ School of Engineering Science, University of Chinese Academy of Sciences, Beijing 100049, China \\ ${ }^{3}$ Department of Mechanics and Aerospace Engineering, Southern University of Science and Technology, Shenzhen, Guangdong 518055, China
}

(Received 11 October 2018; revised manuscript received 29 January 2019; published 27 March 2019)

\begin{abstract}
Direct numerical simulations of three-dimensional compressible helical turbulence are carried out at a grid resolution of $1024^{3}$ to investigate the effect of pressure, which is important for the joint cascade of kinetic energy and helicity in compressible helical turbulence. The principal finding is that the pressure term of the helicity equation [defined as $\Phi^{H}=p \partial_{i}\left(\omega_{i} / \rho\right)$ ] has a smaller effect on the helicity cascade in the aspect of amplitude and a smaller effective range, which leads to a longer inertial subrange of the helicity cascade, in contrast to a kinetic energy cascade. In addition, we also find that the effective range of $\Phi^{H}$ is concentrated only in large scales statistically, which is similar to the effect of the pressure term of the kinetic energy equation (defined as $\Phi^{E}=p \partial_{i} u_{i}$ ). From the overall sense of the effect of $\Phi^{E}$ and $\Phi^{H}$ on the kinetic energy and the helicity, respectively, both of them play a role of dissipation especially in the compression region. We propose that high enough helicity can affect the process of energy transformation between kinetic energy and internal energy, which means that the absolute local helicity hinders the process of kinetic energy transferring to internal energy, and promotes internal energy transferring to kinetic energy. In addition, $\Phi^{H}$ plays a source role both for positive and negative helicity. We also study the mechanism of cancellations between compression and rarefaction regions, and we find that the impact of a shocklet on the helicity cascade can be ignored statistically.
\end{abstract}

DOI: 10.1103/PhysRevE.99.033114

\section{INTRODUCTION}

Compressible turbulence is a key and complex problem for astrophysical systems, inertial confinement fusion, hypersonic aircraft, and multiphase flows. In contrast to incompressible turbulence, the complexity of compressible turbulence is mainly reflected in the aspects of coupling between the velocity field and the thermodynamic field, interactions among vortices, acoustic waves, shock waves, and expansion-like waves and transformation between kinetic energy and internal energy [1]. The theory of turbulence cascade is limited to fully developed incompressible turbulence with a high enough Reynolds number according to the Richardson-KolmogorovOnsager picture [2]. It is difficult to extend this theory to compressible turbulence due to the variable-density effect in the nonlinear term theoretically. Some experiments and numerical simulations of compressible turbulence have confirmed the same scaling exponent $-5 / 3$, such as the warm interstellar medium [3] and the intracluster medium between galaxies [4]. Recently, however, some results of direct numerical simulation (DNS) for compressible turbulence with a high turbulent Mach number $\left(\mathrm{M}_{t}\right)$ [5-10] also reveal the same scaling exponent $-5 / 3$. Some scientific studies for compressible turbulence involve the conception of scale decomposition [11]. The most important research method for compressible turbulence is "coarse-graining," which was proposed by Aluie [11-14]. He tried to employ this filtering method to extend the theory of turbulence cascade in incompressible turbulence

\footnotetext{
*cpyu@imech.ac.cn
}

to compressible turbulence. The biggest obstacle is the appearance of $\Phi^{E}$ in the governing equation of kinetic energy. He argued that $\Phi^{E}$ decays at a rapid rate and only operates in large scales statistically, and then kinetic energy cascades conservatively, which excludes the effect of $\Phi^{E}$ over the transitional conversion range. According to previous conclusions, it was confirmed that there exists a similarity of turbulence cascade in incompressible and compressible turbulence, and it motivates us to explore the theory of joint turbulence cascade of compressible helical turbulence.

Similar to kinetic energy, helicity is also an inviscid invariance in three-dimensional compressible turbulence. It has numerous interesting properties and has been the subject of a huge number of theoretical, numerical, and experimental investigations in the past few decades [15-27]. It is defined as

$$
H=\int_{V} \boldsymbol{u}(\boldsymbol{x}) \cdot \boldsymbol{\omega}(\boldsymbol{x}) d^{3} \boldsymbol{x},
$$

where $\boldsymbol{u}(\boldsymbol{x})$ is the velocity, $\boldsymbol{\omega}(\boldsymbol{x})$ is the vorticity, and the helicity density is defined as $h=\boldsymbol{u} \cdot \boldsymbol{\omega}$, and it is a pseudoscalar. Helical turbulence occurs frequently in atmospheric and astrophysical physics, and its mathematical properties, which are similar to kinetic energy, provide us with novel insight into the physical mechanism of turbulence.

Many studies have shown that a joint cascade of energy and helicity exists in incompressible helical turbulence. Similar to energy flux in the inertial subrange, helicity flux is also a constant in the inertial subrange [15]. Andre et al. found that the helicity cascade hinders the energy cascade and is carried along locally and linearly by the energy cascade like a passive scalar [18]. Chen et al. revealed that the inertial subrange of the helicity cascade is not shorter than the energy cascade 
through the analysis of net helicity flux, and the helicity flux is even more intermittent than the energy flux through the method of helical wave decomposition [20,21]. Biferale et al. discovered that a stationary inverse energy cascade is $E_{\text {back }}(k) \sim k^{-5 / 3}$ and a pure helicity cascade corresponds to a spectrum with $E_{\text {forw }} \sim k^{-7 / 3}$ [23], and they also found that energy flux is always reversed when mirror symmetry is broken or the relative weights of triadic interactions are altered through creating different novel ways to perform numerical investigations of Navier-Stokes equations (NSEs) [22,24]. Controlling the rate of helicity injection at all scales, Kessar et al. found that energy flux could not be changed even with a high rate of helicity injection, and energy scaling law changes from $E(k) \sim k^{-5 / 3}$ in nonhelical status to $E(k) \sim k^{-7 / 3}$ in maximal helical status [28]. To study the impact of helicity on the energy cascade to small scale, Stepanov et al. discussed various scenarios of direct turbulence cascade, and they found that the helical bottleneck effect would appear in the process of energy cascade [25].

The previous studies on helicity cascade were mainly limited to incompressible helical turbulence, and few attempts have been made to investigate it in compressible helical turbulence. The physical regularity of some extreme natural phenomena with the compressibility effect is attributed to helicity [29,30], and it is vital to explore in depth the joint cascade in compressible turbulence. There exist several factors in compressible turbulence that could affect the joint cascade of kinetic energy and helicity, and the effect of pressure is one of the main factors. In this paper, we try to investigate the effect of pressure in detail.

The role of pressure in turbulence dynamics has attracted a great deal of attention to illustrate some features of turbulent flows. Gotoh et al. found that the contribution of the pressure term is important to the dynamics of longitudinal velocity with large amplitudes by analyzing numerical simulation data at a very large Reynolds number in incompressible turbulence [31]. The effect of pressure on turbulence velocity gradient dynamics was investigated through the inviscid three-dimensional Burgers equation and the restricted Euler equation by Bikkani et al., who determined the direction of energy cascade in terms of the changing rate of the magnitude of the velocity gradient tensor [32]. Pumir et al. found that pressure acts on fluid elements to redistribute kinetic energy very differently in two- and three-dimensional flows, and pressure accelerates the three-dimensional fastest fluid elements [33]. Wilczek et al. linked the role of pressure to actual physical turbulent structures [34], and subsequent research carried out by Lawson et al. and Bechlars et al. revealed that pressure stabilizes rotational motions and allows vortices to exist $[35,36]$. In compressible turbulence, there have also been some attempts to study the effect of pressure Hessian on a velocity gradient tensor [37].

In this paper, we investigate the effect of pressure on the joint cascade of kinetic energy and helicity in compressible helical turbulence. In Sec. II, we introduce some numerical details such as governing equations, the numerical method, and some characteristic parameters regarding our DNS. In Sec. III, we analyze the role of the pressure term in the joint cascade of kinetic energy and helicity. In Sec. IV, we carry out statistical analysis of two pressure terms conditioned on local velocity divergence and local helicity. In Sec. V, the role of a shocklet is investigated, and we propose that there exist cancellations between compression and rarefaction regions. In Sec. VI, we summarize our conclusions.

\section{GOVERNING EQUATIONS, NUMERICAL METHODS, AND SOME CHARACTERISTIC PARAMETERS}

The variables in compressible helical turbulence are normalized by the reference density $\rho_{f}$, velocity $U_{f}$, length $L_{f}$, pressure $p_{f}=\rho_{f} U_{f}^{2}$, temperature $T_{f}$, viscosity $\mu_{f}$, thermal conductivity $\kappa_{f}$, and energy per unit volume $\rho_{f} U_{f}^{2}$, respectively [1]. Three reference governing parameters will appear: the reference Reynolds number $\operatorname{Re} \equiv \rho_{f} U_{f} L_{f} / \mu_{f}$, the reference Mach number $\mathrm{M} \equiv U_{f} / c_{f}$, and the reference Prandtl number $\operatorname{Pr} \equiv \mu_{f} C_{p} / \kappa_{f}$, which is assumed to be equal to 0.7 . In addition, other parameters are the speed of sound $c_{f} \equiv$ $\sqrt{\gamma R T_{f}}$, the specific gas constant $R$, the heat capacity ratio $\gamma \equiv C_{p} / C_{v}$, which is assumed to be equal to 1.4 , the specific heat at constant pressure $C_{p}$, the specific heat at constant volume $C_{v}$, and the parameter $\alpha \equiv \operatorname{Pr} \operatorname{Re}(\gamma-1) \mathrm{M}^{2}$.

The dimensionless NSEs for three-dimensional compressible helical turbulence of ideal gas [38] are

$$
\begin{aligned}
\frac{\partial \rho}{\partial t}+\frac{\partial\left(\rho u_{j}\right)}{\partial x_{j}} & =0 \\
\frac{\partial\left(\rho u_{i}\right)}{\partial t}+\frac{\partial\left(\rho u_{i} u_{j}\right)}{\partial x_{j}} & =-\frac{\partial p}{\partial x_{i}}+\frac{1}{\operatorname{Re}} \frac{\partial \sigma_{i j}}{\partial x_{j}}+F_{i}, \\
\frac{\partial \mathcal{E}}{\partial t}+\frac{\partial\left[(\mathcal{E}+p) u_{j}\right]}{\partial x_{j}}= & \frac{1}{\alpha} \frac{\partial}{\partial x_{j}}\left(\kappa \frac{\partial T}{\partial x_{j}}\right) \\
& +\frac{1}{\operatorname{Re}} \frac{\partial\left(\sigma_{i j} u_{i}\right)}{\partial x_{j}}-\Lambda+F_{j} u_{j},
\end{aligned}
$$

where $\rho$ is the density, $u_{i}$ is the $i$ th component of the velocity vector, $p$ is the pressure, $T$ is the temperature, $\Lambda$ is a cooling function for sustaining a steady state statistically, and $F_{i}$ is the $i$ th component of large-scale force composed of multiparameters controlling kinetic energy and helicity. The ideal-gas equation of state is

$$
p=\frac{\rho T}{\gamma M^{2}} .
$$

In addition, the viscous stress is

$$
\sigma_{i j}=\mu\left(\frac{\partial u_{i}}{\partial x_{j}}+\frac{\partial u_{j}}{\partial x_{i}}\right)-\frac{2}{3} \mu \frac{\partial u_{i}}{\partial x_{i}} \delta_{i j}
$$

and the total energy is

$$
\mathcal{E}=\frac{p}{\gamma-1}+\frac{1}{2} \rho\left(u_{i} u_{i}\right) .
$$

The viscosity coefficient $\mu$ and the thermal conductivity coefficient $\kappa$ are determined by the Sutherland law [39] for simplicity,

$$
\begin{aligned}
& \mu=\frac{1.4042 T^{1.5}}{T+0.40417}, \\
& \kappa=\frac{1.4042 T^{1.5}}{T+0.40417} .
\end{aligned}
$$


DNS for forced compressible helical turbulence in a cubic box of $(2 \pi)^{3}$ with periodic boundary conditions is carried out. The initial density field is homogeneous and set to a constant of 1 , and the velocity field is initialized with a radial spectrum $k^{2} \exp \left[-2\left(k / k_{0}\right)^{2}\right]$. Our numerical statistical analysis begins at $t / t_{\text {eddy }}=6.6$ when the skewness of flow is less than -0.5 and total kinetic energy and helicity approach two constants, where $t_{\text {eddy }}$ is the initial large-eddy turnover time. A multiparameter controlling method for external force is proposed for obtaining steady flows with high mean helicity. Stationary injection rates of kinetic energy and helicity are applied within the lowest two wave-number shells in spectral space. A thermal cooling function $\Lambda$ is applied for a steady flow with the formula $\Lambda=\sigma_{I} T^{0}$ [38].

A hybrid compact-WENO scheme [38] is applied in the numerical simulations, which employs a seventh-order WENO scheme [40] in shock regions with a criterion defined as local dilatation $\left(\theta=\partial u_{i} / \partial x_{i}\right)$ for $\theta<-R_{\theta} \theta^{\prime}$, where $R_{\theta}=3.0$ [1] and an eighth-order compact finite-difference scheme [41] in smooth regions. An explicit low storage second-order RungeKutta technique is adopted for time marching. The following numerical results are time-averaged over 20 instantaneous flows from $t / t_{\text {eddy }}=6.6$ to 7.5 .

Next, we would like to introduce some basic characteristic parameters for our numerical simulations [1]. Some characteristic lengths are defined as

$$
\begin{gathered}
L_{f}=\frac{3 \pi}{2 u^{\prime 2}} \int_{0}^{\infty} \frac{E(k)}{k} d k, \\
\lambda=\frac{u^{\prime}}{\left\langle\left[\left(\partial u_{1} / \partial x_{1}\right)^{2}+\left(\partial u_{2} / \partial x_{2}\right)^{2}+\left(\partial u_{3} / \partial x_{3}\right)^{2}\right]\right\rangle^{1 / 2}},
\end{gathered}
$$

and

$$
\eta=\left[\langle\mu / \operatorname{Re} \rho\rangle^{3} / \epsilon\right]^{1 / 4},
$$

where $L_{f}$ is the longitudinal integral length scale, $\lambda$ is the transverse Taylor microscale, and $\eta$ is the Kolmogorov length scale. Here, $E(k)$ is the spectrum of kinetic energy per unit mass, $u^{\prime}$ is the root mean square (rms represented by a prime) of velocity defined as $u^{\prime}=\sqrt{u_{1}^{2}+u_{2}^{2}+u_{3}^{2}}$, and $\epsilon$ is the ensemble-averaged viscous dissipation rate of kinetic energy per unit volume defined as $\epsilon=\left\langle\sigma_{i j} S_{i j} /(\operatorname{Re} \rho)\right\rangle$, where the strain-rate tensor is defined as $S_{i j}=(1 / 2)\left(\partial u_{i} / \partial x_{j}+\right.$ $\left.\partial u_{j} / \partial x_{i}\right)$.

The Taylor microscale Reynolds number and turbulence Mach number are

$$
\operatorname{Re}_{\lambda}=\frac{u^{\prime} \lambda\langle\rho\rangle}{\sqrt{3}\langle\mu\rangle}
$$

and

$$
\mathrm{M}_{t}=\mathrm{M} \frac{u^{\prime}}{\langle\sqrt{T}\rangle} .
$$

In this paper, Helmholtz decomposition and helical wave decomposition are used to analyze the DNS data. Both of them provide two orthogonal basis vectors to decompose a three-dimensional flow field. Helmholtz decomposition is widely used in the area of compressible turbulence to decompose velocity into compressive and rotational components.
The compressive component is rotation-free $\left(i \mathbf{k} \times \mathbf{V}^{c}=\mathbf{0}\right)$, and the rotational component is divergence-free $\left(i \mathbf{k} \cdot \mathbf{V}^{r}=\mathbf{0}\right)$. They are

$$
\begin{aligned}
\mathbf{V}^{c}(\mathbf{k}) & =\frac{\mathbf{k}}{k^{2}}(\mathbf{k} \cdot \mathbf{V})(\mathbf{k}), \\
\mathbf{V}^{r}(\mathbf{k}) & =\mathbf{V}(\mathbf{k})-\mathbf{V}^{c}(\mathbf{k}),
\end{aligned}
$$

where superscripts $c$ and $r$ represent the compressive and rotational components, respectively [42]. Helical wave decomposition has been popular for projecting velocity on a pair of orthogonal bases formed by $\mathbf{h}^{+}$and $\mathbf{h}^{-}[43,44]$. The "inertial wave" forms are

$$
\mathbf{h}^{s}=\boldsymbol{v} \times \boldsymbol{\kappa}+i s \boldsymbol{v},
$$

where $\boldsymbol{v}$ is a unit vector orthogonal to $\mathbf{k}$, and an alternative form is $\boldsymbol{v}=(\mathbf{z} \times \boldsymbol{\kappa}) /\|\mathbf{z} \times \boldsymbol{\kappa}\|$. Here $\mathbf{k}=k \boldsymbol{\kappa}, \boldsymbol{\kappa}$ is the unit vector of wave-number vector $\boldsymbol{k}$, and $\mathbf{z}$ is an arbitrary vector. Therefore, we can expand the velocity as

$$
\begin{aligned}
\mathbf{u}(\mathbf{x}) & =\sum_{\mathbf{k}} \mathbf{u}(\mathbf{k}) e^{i \mathbf{k} \cdot \mathbf{x}} \\
& =\sum_{\mathbf{k}}\left(\mathbf{u}^{+}+\mathbf{u}^{c}+\mathbf{u}^{-}\right) e^{i \mathbf{k} \cdot \mathbf{x}},
\end{aligned}
$$

where right-chirality velocity is defined as $\mathbf{u}^{+}=a^{+} \mathbf{h}^{+}$, leftchirality velocity is defined as $\mathbf{u}^{-}=a^{-} \mathbf{h}^{-}$, and the compressive component of velocity is called free-chirality velocity $\mathbf{u}^{c}$. Here $a^{+}$and $a^{-}$are the projections of velocity on $\mathbf{h}^{+}$ and $\mathbf{h}^{-}$. The basis vectors $\mathbf{h}^{s}(s= \pm 1)$ could be complex and orthogonal to $\mathbf{k}$ and to each other. The useful property of these basis vectors is that they are the eigenmodes of the curl operator:

$$
i \mathbf{k} \times \mathbf{h}^{s}=s k \mathbf{h}^{s} .
$$

In general, we can combine Helmholtz decomposition and helical wave decomposition to propose a uniform decomposition, which is called general helical wave decomposition (GHWD) of compressible turbulence in this paper. This new uniform method of decomposition could be generalized from helical wave decomposition, and the superscript $s=0$ corresponding to the compressive component of velocity is added in Eq. (17), in contrast to traditional helical wave decomposition $(s=-1,1)$. Hence we extend helical wave decomposition to GHWD, which provides three orthogonal basis vectors in compressible turbulence. Here, $s=-1$ corresponds to left chirality, $s=0$ corresponds to free chirality, and $s=+1$ corresponds to right chirality. For simplicity in this paper, we use the notation $s=-, 0,+$, respectively. The kinetic energy content of three modes is

$$
E^{s}(k)=\sum_{k \leqslant|\mathbf{k}|<k+1} \frac{1}{2}\left|u_{\mathbf{k}}^{s}\right|^{2}, s=-, 0,+.
$$

If there exists a joint cascade of energy and helicity and the distortion time is the same for both energy and helicity transfer, we can obtain the power-law solutions, which ignore any intermittency corrections in incompressible 

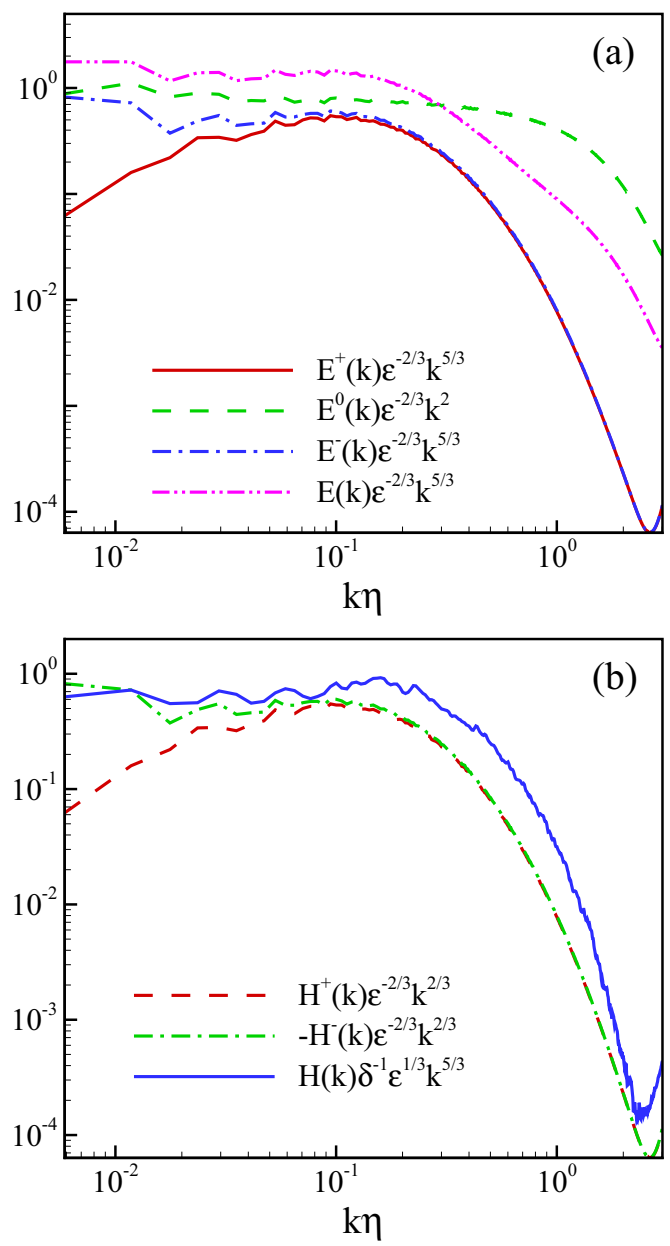

FIG. 1. (a) Compensated spectra of right-chirality kinetic energy (solid red line), free-chirality kinetic energy (dashed green line), left-chirality kinetic energy (blue dot-dashed line), and total kinetic energy (double dot-dashed pink line). (b) Compensated spectra of right-chirality helicity (dashed red line), left-chirality helicity (dotdashed green line), and total helicity (solid blue line).

turbulence [15,20,45],

$$
\begin{gathered}
E(k) \sim C_{E} \epsilon^{2 / 3} k^{-5 / 3}, H(k) \sim C_{H} \delta \epsilon^{-1 / 3} k^{-5 / 3}, \\
E^{ \pm}(k) \sim \frac{1}{2} \epsilon^{2 / 3} k^{-5 / 3}\left[1 \pm \frac{\beta}{2 k}\left(\frac{\delta}{\epsilon}\right)\right], \\
H^{ \pm}(k) \sim \epsilon^{2 / 3} k^{-2 / 3}\left[1 \pm \frac{\beta}{2 k}\left(\frac{\delta}{\epsilon}\right)\right],
\end{gathered}
$$

where $C_{E}$ and $C_{H}$ are two constants, $\beta=C_{H} / C_{E}, \delta$ is the ensemble-averaged viscous dissipation rate of helicity per unit volume defined as $\delta=\left\langle\mu / \operatorname{Re} S_{i j} R_{i j}\right\rangle$, and $R_{i j}=(1 / 2)\left(\partial \omega_{i} / \partial x_{j}+\partial \omega_{j} / \partial x_{i}\right)$. And the theory of threedimensional Burgers turbulence reveals that shock waves lead to a $k^{-2}$ spectrum of $\mathbf{u}^{0}$ [46].

According to the above-mentioned definitions of characteristic parameters, we exhibit some results in Fig. 1, Fig. 2, and Table I. In Fig. 1(a), we exhibit compensated spectra of left-chirality and right-chirality kinetic energy normalized by $\epsilon^{-2 / 3} k^{5 / 3}$, free-chirality kinetic energy normalized by $\epsilon^{-2 / 3} k^{2}$,

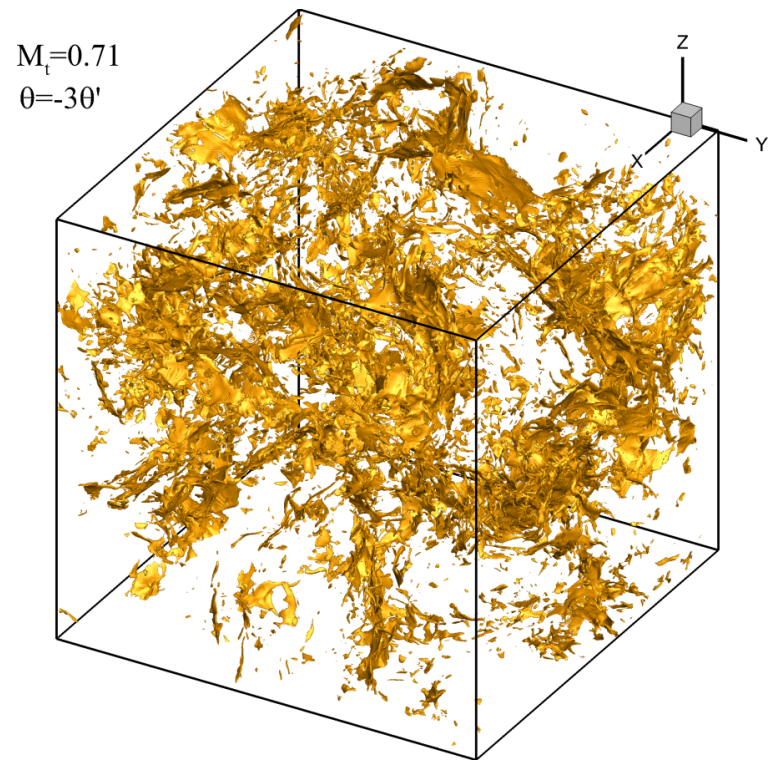

FIG. 2. Isosurface of the velocity divergence at $\theta=-3 \theta^{\prime}$ of the $1024^{3}$ simulation at $M_{t}=0.71$.

and total kinetic energy normalized by $\epsilon^{-2 / 3} k^{5 / 3}$ according to Eqs. (19) and (20). In our numerical simulations, the Reynolds number is high enough to show a visual inertial subrange represented by a large plateau range. The discrepancy of $E^{+}(k)$ and $E^{-}(k)$ is large, especially in a low wave number, to ensure a left-chirality dominant flow field. Compensated spectra of negative left-chirality helicity normalized by $\epsilon^{-2 / 3} k^{2 / 3}$, rightchirality helicity normalized by $\epsilon^{-2 / 3} k^{2 / 3}$, and total helicity normalized by $\delta^{-1} \epsilon^{1 / 3} k^{5 / 3}$ are shown in Fig. 1(b) according to Eqs. (19) and (21), and they also exhibit a large plateau range. We display an isosurface of the velocity divergence at $\theta=-3 \theta^{\prime}$ in Fig. 2, where $\theta^{\prime}$ is the rms of $\theta$. A great deal of stochastic sheetlike shocklets are visible to the naked eye. Shocklets that are present in compressible helical turbulence are produced by the fluctuating field of turbulent eddies [47], and their properties and features regarding jumps of pressure, density, and temperature are similar to the typical shock that is pivotal in high-speed flight. High enough $k_{\max } \eta$ and $O(\eta / \Delta x) \sim 1$ in Table I ensure a fine resolution to resolve the Kolmogorov length scale. The rate of mean kinetic energy and mean helicity guarantees that the helicity cascade does indeed exist.

\section{THE ROLE OF THE PRESSURE TERM IN THE JOINT CASCADE OF KINETIC ENERGY AND HELICITY}

For the purpose of studying the dynamical behavior of turbulence cascade in the inertial subrange of compressible helical turbulence, we need a proper method of scale decomposition and a proper definition of large-scale kinetic energy.

TABLE I. Some characteristic parameters of numerical simulations.

\begin{tabular}{lcccccccc}
\hline \hline Resolution & $\mathrm{Re}_{\lambda}$ & $\mathrm{M}_{t}$ & $E$ & $H$ & $k_{\max } \eta$ & $\eta / \Delta x$ & $L_{f} / \eta$ & $\lambda / \eta$ \\
\hline $1024^{3}$ & 232 & 0.71 & 2.56 & -2.36 & 3.01 & 0.96 & 205 & 30 \\
\hline \hline
\end{tabular}


Recently, Aluie proposed that a Favre decomposition satisfies the inviscid criterion in compressible turbulence $[11,13,14]$. "Coarse graining" is popular for scale decomposition to understand scale interactions in compressible turbulence. The coarse-grained filtered field for any physical variable $\mathbf{a}(\mathbf{x})$ is defined as

$$
\overline{\mathbf{a}}_{l}(\mathbf{x})=\int d^{3} \mathbf{r} G_{l}(\mathbf{r}) \mathbf{a}(\mathbf{x}+\mathbf{r}),
$$

which contains modes larger than filter width $l$, and $G_{l}(\mathbf{r})$ is a normalized convolution kernel. With the help of "coarsegraining," we can obtain coarse-grained field $\overline{\mathbf{a}}_{l}$ and a residual field $\mathbf{a}^{\prime}$, where $\mathbf{a}^{\prime}=\mathbf{a}-\overline{\mathbf{a}}_{l}$. In this paper, we employ a tophat filter to obtain a coarse-grained field in our numerical simulations as follows [9]:

$$
\bar{a}_{i}=\frac{1}{4 n}\left(a_{i-n}+2 \sum_{j=i-n+1}^{i+n-1} a_{j}+a_{i+n}\right),
$$

where the filter width is $l=2 n \Delta x$, and $\Delta x$ is the grid scale in our simulations.

Favre filtering is widely used in the area of large-eddy simulations (LES) of compressible flows. Mathematically, any scalar or vector weighted by density could be rewritten as [48]

$$
\tilde{a}_{l}=\frac{{\overline{(\rho a)_{l}}}_{l}}{\bar{\rho}_{l}},
$$

where $l$ is the filter width. The main advantage of Favre filtering is that the "Favre-filtered" equations are structurally similar to their corresponding nonfiltered equations. This similarity provides much convenience for studying subgrid kinetic energy flux along different scales.

In the governing equation of kinetic energy in decaying incompressible homogeneous and isotropic turbulence, the only source of kinetic energy is the viscosity term, which diffuses and dissipates kinetic energy via molecular viscosity. In compressible turbulence, the source of kinetic energy is not only the viscosity term, but also $\Phi^{E}$, which transforms energy between kinetic energy and internal energy. If we define $\varepsilon_{k}^{i}=$ $u_{i} u_{i} / 2$ for kinetic energy in incompressible turbulence and $\varepsilon_{k}^{c}=\rho u_{i} u_{i} / 2$ for kinetic energy in compressible turbulence, these ensemble-averaged equations are

$$
\begin{gathered}
\frac{\partial\left\langle\varepsilon_{k}^{i}\right\rangle}{\partial t}=-\left\langle\sigma_{i j}^{i} \frac{\partial u_{i}}{\partial x_{j}}\right\rangle, \\
\frac{\partial\left\langle\varepsilon_{k}^{c}\right\rangle}{\partial t}=\langle p \theta\rangle-\left\langle\sigma_{i j}^{c} \frac{\partial u_{i}}{\partial x_{j}}\right\rangle,
\end{gathered}
$$

where $\sigma_{i j}^{i}=\mu\left(\partial u_{i} / \partial x_{j}+\partial u_{j} / \partial x_{i}\right)$ for incompressible turbulence, $\sigma_{i j}^{c}=\mu\left(\partial u_{i} / \partial x_{j}+\partial u_{j} / \partial x_{i}\right)-2 / 3 \mu \theta \delta_{i j}$ for compressible turbulence, and the symbols $i$ and $c$ correspond to incompressible turbulence and compressible turbulence, respectively.

The biggest discrepancy in the above two equations lies in the pressure term, which turns out to be an obstacle for extending the theory of turbulence cascade in incompressible turbulence to compressible turbulence. Fortunately, Aluie proved that the pressure term for kinetic energy operates only in large scales, which lie in the transitional "conversion" scale range statistically [14]. If the effect of the pressure term were avoided statistically in the kinetic energy equation, kinetic energy would cascade conservatively in the inertial subrange of compressible turbulence in analogy with incompressible turbulence. The uniform formalism of the governing equation makes it somewhat convenient to research issues of locality in compressible turbulence [49].

Similar to the energy cascade in compressible turbulence, there also exists the helicity cascade in compressible turbulence from the standpoint of the property of the governing equation of helicity. The identical challenge faced in analyzing the helicity cascade in the area of compressible turbulence is also the effect of $\Phi^{H}$, hence in this section we want to study the effect of $\Phi^{H}$ by the method used in $\Phi^{E}$.

Now, we review the ensemble-averaged governing equations of kinetic energy and helicity in compressible turbulence. In what follows, all objects are compressible turbulence. We define $\varepsilon=\rho u_{i} u_{i} / 2$ for kinetic energy and $H=u_{i} \omega_{i}$ for helicity density. Then the ensemble-averaged equations are

$$
\begin{gathered}
\frac{\partial\langle\varepsilon\rangle}{\partial t}=\left\langle\Phi^{E}\right\rangle-\left\langle\sigma_{i j} \frac{\partial u_{i}}{\partial x_{j}}\right\rangle \\
\frac{\partial\langle H\rangle}{\partial t}=\left\langle 2 \Phi^{H}\right\rangle-\left\langle 2 \sigma_{i j} \frac{\partial\left(\omega_{i} / \rho\right)}{\partial x_{j}}\right\rangle .
\end{gathered}
$$

The first term $\left\langle 2 \Phi^{H}\right\rangle$ on the right-hand side of the helicity equation does not exist in incompressible turbulence because of the constant density. After comparing $\Phi^{E}$ and $\Phi^{H}$, we were surprised to find that they are similar in structure, and both of them involve the effect of variable density. In the following, we will extend the researching method of $\Phi^{E}$ to $\Phi^{H}$.

The definitions of the pressure-dilatation cospectrum [13] in the kinetic energy equation (PD) and the pressure-vorticity per mass cospectrum in the helicity equation (PV) are

$$
\begin{gathered}
E^{\mathrm{PD}}(k)=\sum_{k-0.5<|\mathbf{k}|<k+0.5}-\widehat{p}(\mathbf{k}) \widehat{\nabla \cdot \mathbf{u}}(-\mathbf{k}), \\
\left.E^{\mathrm{PV}}(k)=\sum_{k-0.5<|\mathbf{k}|<k+0.5}-\widehat{p}(\mathbf{k}) \nabla \widehat{(\boldsymbol{\omega} / \rho} \rho\right)(-\mathbf{k}) .
\end{gathered}
$$

If they decay fast enough along the wave number,

$$
\begin{gathered}
\left|E^{\mathrm{PD}}(k)\right| \leqslant C_{1} u_{\mathrm{rms}} p_{\mathrm{rms}}\left(k L_{f}\right)^{-\beta_{1}}, \beta_{1}>1, \\
\left|E^{\mathrm{PV}}(k)\right| \leqslant C_{2}(\omega / \rho)_{\mathrm{rms}} p_{\mathrm{rms}}\left(k L_{f}\right)^{-\beta_{2}}, \beta_{2}>1,
\end{gathered}
$$

then the effect of two pressure terms over a transitional conversion scale range would approach a limited extent, where $C_{1}$ and $C_{2}$ are two constants. Beyond this range, kinetic energy and helicity would cascade conservatively in the inertial subrange. Under the above assumptions, ensemble-averaged $\Phi^{E}$ and $\Phi^{H}$ would asymptote to a finite constant with the increase of the filter width,

$$
\begin{gathered}
\operatorname{PD}(l)=-\left\langle\bar{p}_{l} \nabla \cdot \overline{\mathbf{u}}_{l}\right\rangle, \zeta_{1}=-\langle p \nabla \cdot \mathbf{u}\rangle, \\
\operatorname{PV}(l)=-\left\langle\bar{p}_{l} \nabla \cdot \overline{(\boldsymbol{\omega} / \rho)_{l}}\right\rangle, \zeta_{2}=-\langle p \nabla \cdot(\boldsymbol{\omega} / \rho)\rangle,
\end{gathered}
$$

where $\operatorname{PD}(l)$ is the cumulative $\mathrm{PD}$, and $\zeta_{1}$ is an ultimate value for it; $\mathrm{PV}(l)$ is the cumulative $\mathrm{PV}, \zeta_{2}$ is an ultimate value for it, and $l \sim 1 / k$. 


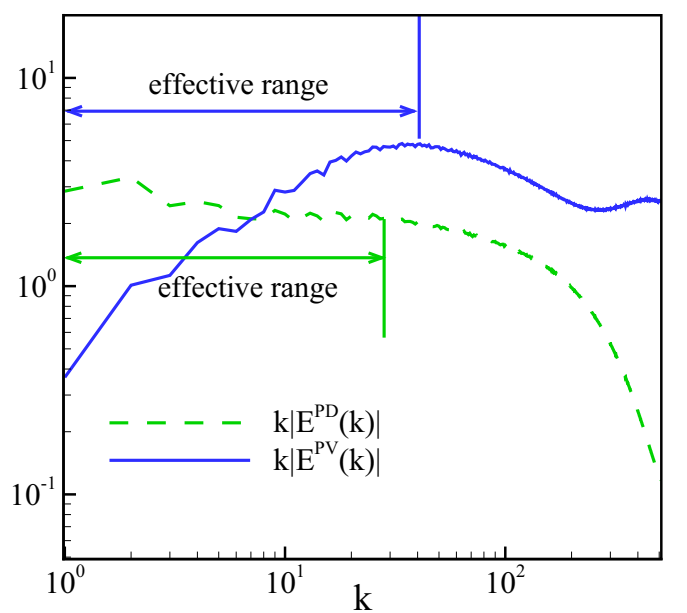

FIG. 3. Compensated pressure-dilatation cospectrum (dashed green line) and compensated pressure-vorticity per mass (solid blue line).

In our numerical simulations, we calculated the absolute value of Eqs. (29) and (30), and we show our results of these two compensated cospectra normalized by $k$ in Fig. 3 . Obviously, both compensated cospectra begin to decay over some critical wave number. This result confirms a previous assumption about scaling of PD, and the reliability of our numerical simulations. While in the range of lower wave number, PV prefers to grow gradually faster than PD, and then it begins to decay over a critical wave number. If we defined a critical wave number where the compensated spectrum begins to decay, the critical wave number for PV is larger than the critical wave number for PD. In other words, the effective range of PV on the helicity cascade is larger than the effective range of PD on the kinetic energy cascade according to assumptions in Eqs. (31) and (32). This phenomenon is seemingly in conflict with the previous opinion. We try to explain that the evaluation in Eqs. (31) and (32) is a sufficient condition for the cumulative effect, and it overestimates the actual effect of $\Phi^{E}$ and $\Phi^{H}$ through calculating the spectrum of the corresponding absolute value. But this method can predict the cumulative trend successfully, and it confirms that the effect of $\Phi^{H}$ on the helicity cascade only concentrates in large scales statistically similar to the effect of $\Phi^{E}$ on the kinetic energy cascade.

To study $\Phi^{E}$ and $\Phi^{H}$ in detail, ensemble-averaged filtered governing equations for kinetic energy and helicity are inferred by Favre filtering as follows. The ensemble-averaged filtered kinetic energy equation is

$$
\partial_{t}\left\langle\bar{\rho}_{l} \frac{\left|\widetilde{\mathbf{u}}_{l}\right|^{2}}{2}\right\rangle=-\left\langle\Pi_{l}^{E}\right\rangle-\left\langle\Lambda_{l}\right\rangle+\left\langle\Phi_{l}^{E}\right\rangle-\left\langle D_{l}^{E}\right\rangle+\left\langle\varepsilon_{l}^{\mathrm{inj}}\right\rangle,
$$

where some terms in this equation are defined as

$$
\begin{aligned}
\Pi_{l}^{E} & =-\bar{\rho}_{l} \partial_{j} \tilde{u}_{i} \tilde{\tau}\left(u_{i}, u_{j}\right), \\
\Lambda_{l} & =\frac{1}{\bar{\rho}_{l}} \partial_{j} \bar{p}_{l}\left(\overline{\rho u_{j}}-\bar{u}_{j} \bar{\rho}_{l}\right), \\
\Phi_{l}^{E} & =\bar{p}_{l} \nabla \cdot \overline{\mathbf{u}}_{l},
\end{aligned}
$$
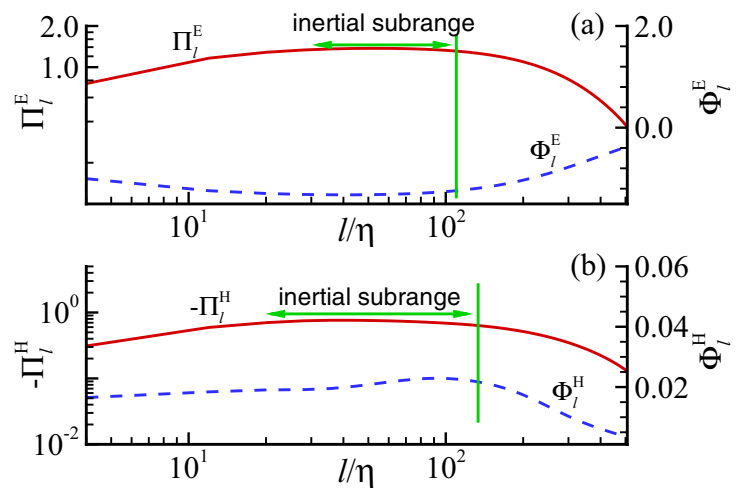

FIG. 4. (a) Ensemble-averaged subgrid kinetic energy flux $\Pi_{l}^{E}$ (solid red line), and ensemble-averaged pressure term $\Phi_{l}^{E}$ (dashed blue line) in the kinetic energy equation. (b) Ensemble-averaged negative subgrid helicity flux $-\Pi_{l}^{H}$ (solid red line), and ensembleaveraged pressure term $\Phi_{l}^{H}$ (dashed blue line) in the helicity equation, where $l$ is the filter width, and $\eta$ is the Kolmogorov dissipation length scale.

$$
\begin{aligned}
D_{l}^{E} & =\partial_{j} \widetilde{u}_{i}\left(2 \overline{\mu S_{i j}}-\frac{2}{3} \overline{\mu S_{k k}} \delta_{i j}\right), \\
\varepsilon_{l}^{\mathrm{inj}} & =\widetilde{u}_{i} \bar{\rho}_{l} \widetilde{F}_{i},
\end{aligned}
$$

$\Pi_{l}^{E}$ is the kinetic energy flux due to subgrid scale stress, $\Lambda_{l}$ is the kinetic energy flux due to turbulent mass flux, $\Phi_{l}^{E}$ is the filtered pressure term, which is consistent with the definition $\Phi^{E}, D_{l}^{E}$ is the viscous dissipation, $\varepsilon_{l}^{\text {inj }}$ is the work of external force, and the superscript $E$ denotes kinetic energy.

The ensemble-averaged filtered helicity equation is

$$
\partial_{t}\langle\widetilde{H}\rangle=-\left\langle\Pi_{l}^{H}\right\rangle+\left\langle\Phi_{l}^{H}\right\rangle-\left\langle D_{l}^{H}\right\rangle+\left\langle\eta_{l}^{\mathrm{inj}}\right\rangle,
$$

where some terms in this equation are defined as

$$
\begin{aligned}
\widetilde{H} & =\widetilde{u}_{i} \cdot \widetilde{\omega}_{i}, \\
\Pi_{l}^{H} & =-\bar{\rho}_{l} \partial_{j}\left(2 \widetilde{\omega}_{i} / \bar{\rho}_{l}\right) \widetilde{\tau}\left(u_{i}, u_{j}\right), \\
\Phi_{l}^{H} & =\bar{p}_{l} \partial_{i}\left(2 \widetilde{\omega}_{i} / \bar{\rho}_{l}\right), \\
D_{l}^{H} & =\partial_{j}\left(2 \widetilde{\omega}_{i} / \bar{\rho}_{l}\right)\left(2 \overline{\mu S_{i j}}-\frac{2}{3} \overline{\mu S_{k k}} \delta_{i j}\right), \\
\eta_{l}^{\text {inj }} & =2 \widetilde{\omega}_{i} \widetilde{F}_{i},
\end{aligned}
$$

$\tilde{H}$ is the filtered helicity density, $\Pi_{l}^{H}$ is the helicity flux due to subgrid scale stress, $\Phi_{l}^{H}$ is the filtered pressure term, which is consistent with the definition $\Phi^{H}, D_{l}^{H}$ is the viscous dissipation, $\eta^{\mathrm{inj}}$ is the work of external force, and superscript $H$ denotes helicity. The notation we employ in the above equations is $\widetilde{\tau}\left(u_{i}, u_{j}\right)=\widetilde{u_{i} u_{j}}-\widetilde{u}_{i} \widetilde{u}_{j}$.

We try to explain in detail the effect of $\Phi_{l}^{E}$ on the kinetic energy cascade and the effect of $\Phi_{l}^{H}$ on the helicity cascade from the standpoint of filtered governing equations. Filtered results of different filter width are shown in Figs. 4(a) and 4(b), and they share a number of similar features. First, both of them exhibit an inertial subrange, which is marked by a green double-headed arrow. Second, the ensemble-averaged values of pressure terms begin to increase and then reach an ultimate value with the decrease of the filter width. There 

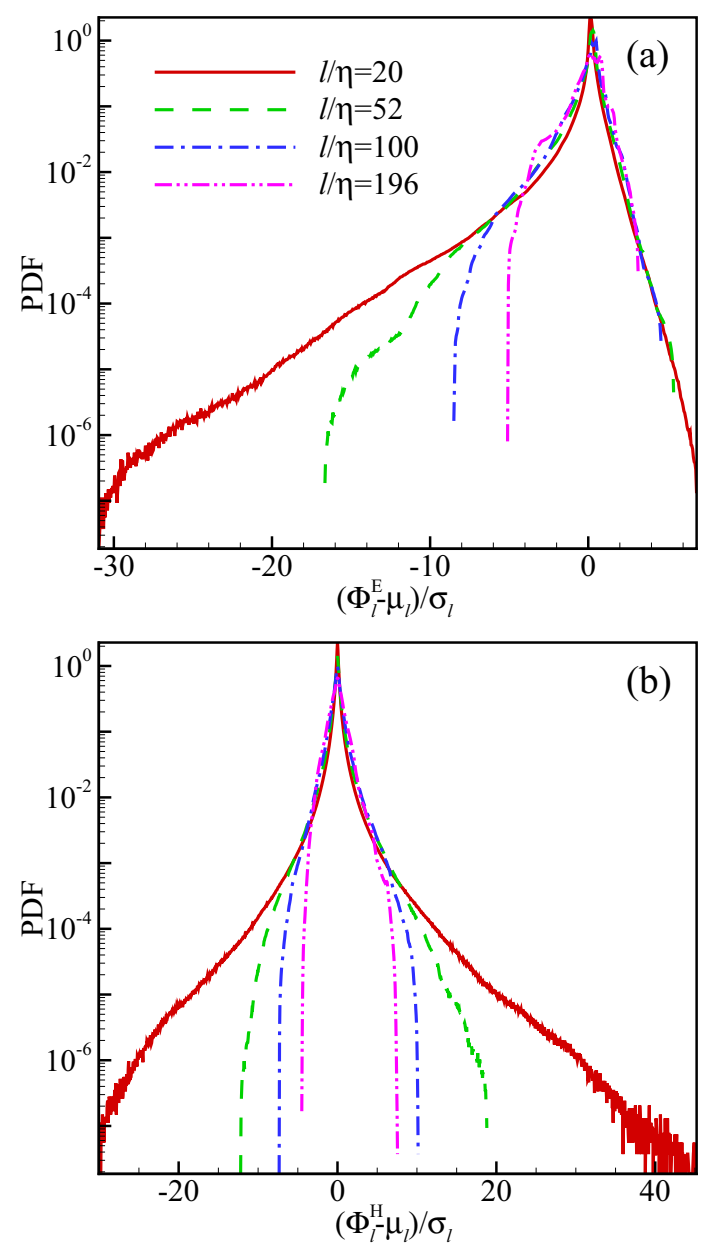

FIG. 5. (a) Normalized pressure term for kinetic energy of different filter width. (b) Normalized pressure term for helicity of different filter width.

exists a critical filter width that splits out the effective range under pressure for the kinetic energy cascade and the helicity cascade. If the filter width is less than the critical filter width, the kinetic energy or the helicity will cascade conservatively, escaping from the effect of the pressure term.

Apparently, the critical filter width of $\Phi_{l}^{E}$ is smaller than the critical filter width of $\Phi_{l}^{H}$, which is obvious in Figs. 4(a) and 4(b). This means that the effective range of $\Phi_{l}^{H}$ is smaller and the inertial subrange of the helicity cascade is longer, while the ratio of the pressure term and the subgrid term for helicity is less than $10 \%$, and the influence of $\Phi_{l}^{H}$ on the helicity cascade is less than the influence of $\Phi_{l}^{E}$ on the kinetic energy cascade.

The probability distribution function (PDF) of normalized $\Phi_{l}^{E}$ and $\Phi_{l}^{H}$ with a different filter width is exhibited in Fig. 5. The filter width $l=20 \eta$ corresponds to the dissipation range, $l=52 \eta$ and $100 \eta$ corresponds to the inertial subrange, and $l=196 \eta$ corresponds to the transitional conversion range. The PDF of $\Phi_{l}^{E}$ is skewed clearly to a negative value, and the fluctuating amplitude is mainly decreased in the negative branch with the increase of the filter width. As for $\Phi_{l}^{H}$, there does not exist an obvious skewness to the negative or positive value, which leads to a small mean but a large
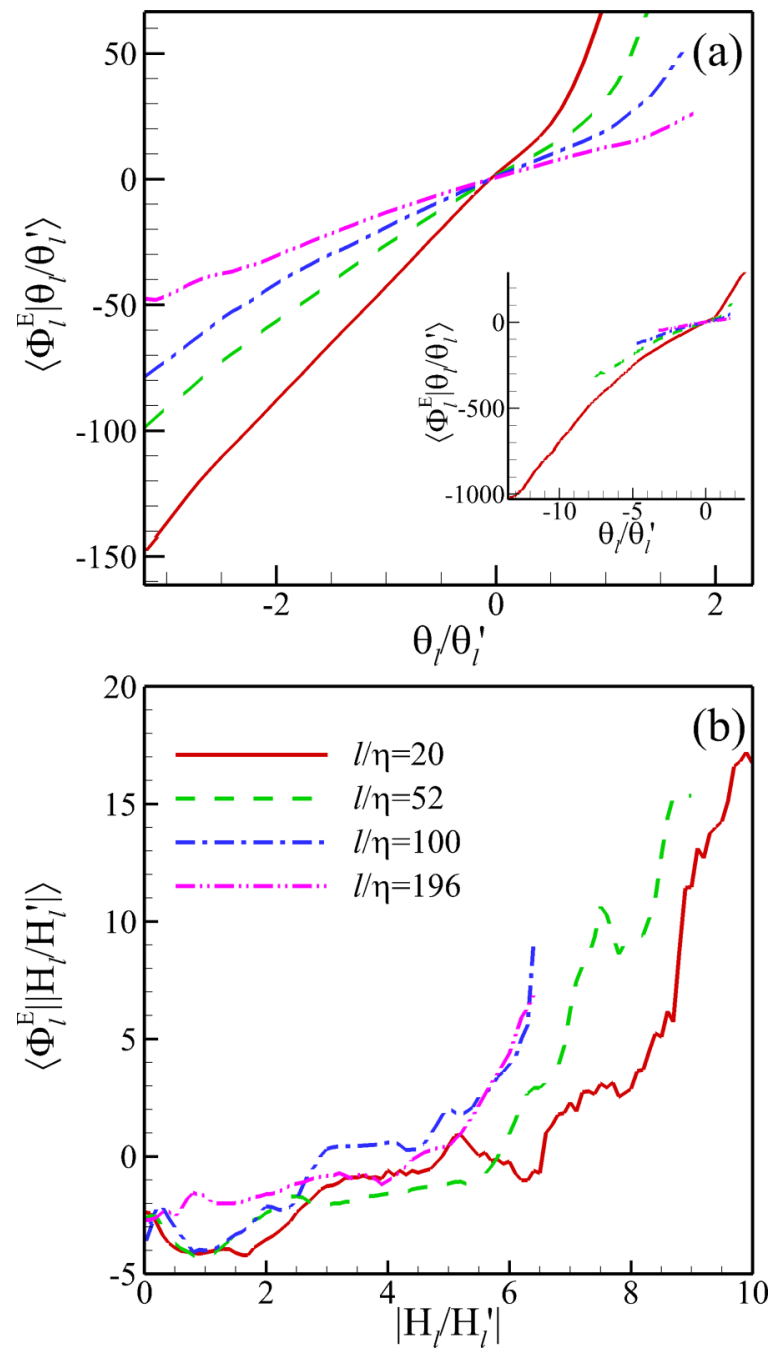

FIG. 6. (a) Ensemble-averaged $\Phi_{l}^{E}$ conditioned on local velocity divergence with a different filter width. (b) Ensemble-averaged $\Phi_{l}^{E}$ conditioned on absolute local helicity with a different filter width.

variation of $\Phi_{l}^{H}$. With the increase of the filter width, the fluctuating amplitude is decreased both in the negative and the positive branch simultaneously. This regularity of distribution illustrates the reason why $k\left|E^{\mathrm{PV}}(k)\right|$ is larger and decays more slowly than $k\left|E^{\mathrm{PD}}(k)\right|$ in Fig. 3. Figure 3 focuses on the spectrum of absolute values, and Fig. 4 focuses on the net contribution of total values. In the process of calculating the net contribution, there is a cancellation between positive and negative fluctuating values, but the fluctuating contribution is reflected in the spectrum of absolute values.

Hence we conclude that the upper bound of the inertial subrange for kinetic energy is less than the helicity. For incompressible turbulence, Ditlevsen et al. proposed that helicity flux is not a constant up to a smaller wave number than energy flux both in the right-hand mode and the left-hand mode [50,51]. But Chen et al. argued that the inertial subrange of the helicity cascade is not shorter than that of the kinetic energy cascade [20]. All of them tried to compare the lower bound for kinetic energy and the helicity cascade, while in our research we prefer to determine their upper bound under the effect of 

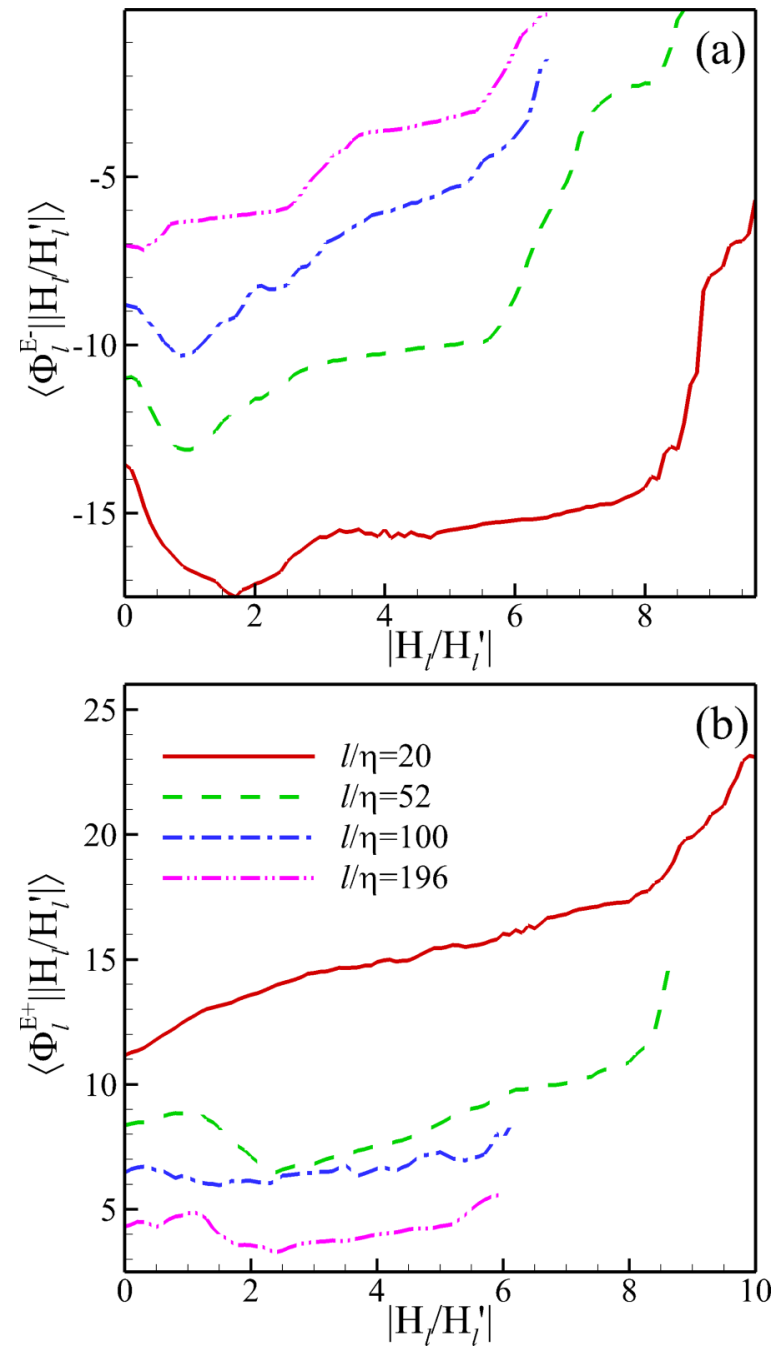

FIG. 7. (a) Ensemble-averaged negative $\Phi_{l}^{E}$ conditioned on absolute local helicity with a different filter width. (b) Ensembleaveraged positive $\Phi_{l}^{E}$ conditioned on absolute local helicity with a different filter width.

the pressure term statistically. Therefore, we can conclude that the inertial subrange of the kinetic energy cascade is shorter than that of the helicity cascade in compressible helical turbulence.

\section{STATISTICAL ANALYSIS OF TWO PRESSURE TERMS CONDITIONED ON LOCAL VELOCITY DIVERGENCE AND LOCAL HELICITY}

The gradient of the inverse of the density will increase to some extreme value at some local area with strong compressibility. We infer that these two pressure terms are both sensitive to the presence of a shocklet, which is represented by velocity divergence.

We analyze ensemble-averaged $\Phi_{l}^{E}$ conditioned on local velocity divergence and absolute local helicity with a different filter width in Fig. 6. Obviously, the ensemble-averaged $\Phi_{l}^{E}$ grows monotonously with the increase of local velocity divergence in Fig. 6(a). With the increase of the filter width, the ensemble-averaged value will decrease because the
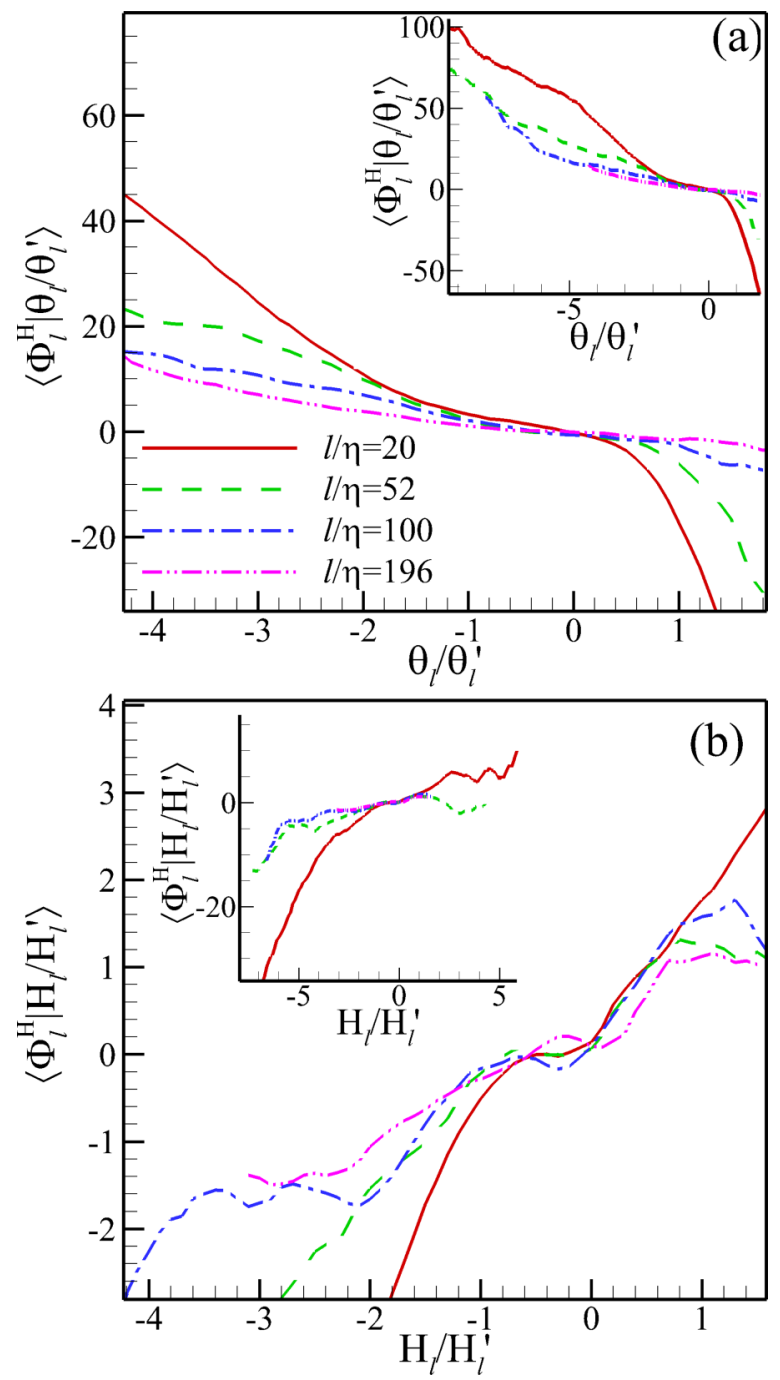

FIG. 8. (a) Ensemble-averaged $\Phi_{l}^{H}$ conditioned on local velocity divergence with a different filter width. (b) Ensemble-averaged $\Phi_{l}^{H}$ conditioned on local helicity with a different filter width.

fluctuation of small scales is stronger than that of large scales. While ensemble-averaged $\Phi_{l}^{E}$ conditioned on absolute local helicity in Fig. 6(b) behaves differently, it begins to increase monotonously from a negative value to a positive value with the increase of absolute local helicity when absolute local helicity is high enough. Positive $\Phi_{l}^{E}$ corresponds to the process of internal energy transferring to kinetic energy, and negative $\Phi_{l}^{E}$ corresponds to the process of kinetic energy transferring to internal energy under the effect of pressure. We find that high enough absolute local helicity could affect the energy conversion process in Fig. 6(b). To further confirm the physical process of energy conversion, we show the ensembleaveraged positive and negative $\Phi_{l}^{E}$ conditioned on absolute local helicity in Fig. 7. When absolute local helicity is high enough, the process of internal energy transferring to kinetic energy $\Phi_{l}^{E+}$ is promoted while the inverse process $\Phi_{l}^{E-}$ is hindered. From that point of view, we can conclude that high enough helicity could decrease the loss of kinetic energy and increase the efficiency of energy conversion from internal energy to kinetic energy through the pressure term. 

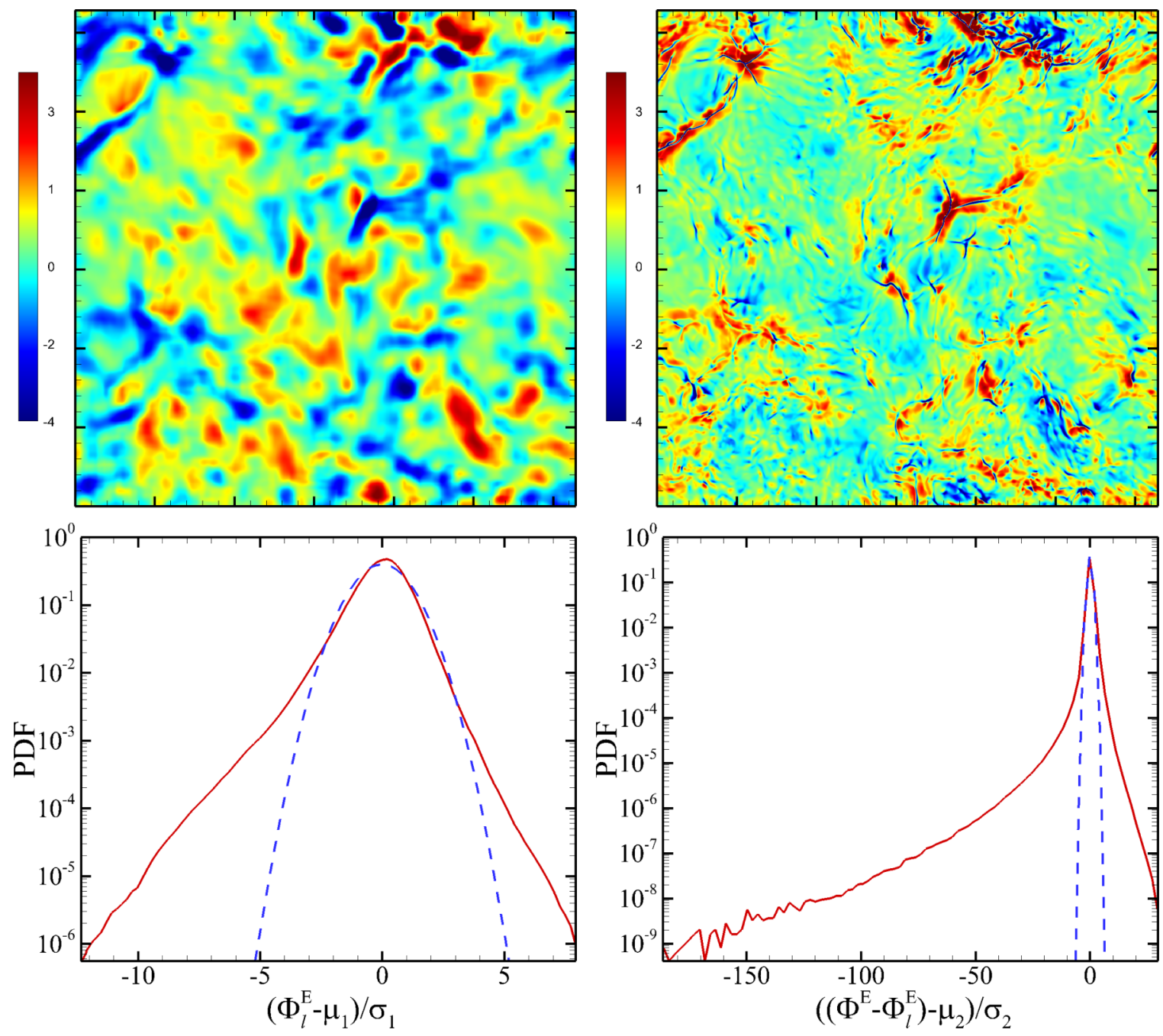

FIG. 9. Top left: two-dimensional slice of pointwise $\Phi_{l}^{E}$. Bottom left: normalized distribution curve for $\Phi_{l}^{E}$ (red solid line) and the corresponding Gaussian distribution (blue dashed line). Top right: two-dimensional slice of residual pointwise $\Phi_{l}^{E}$. Bottom right: normalized distribution curve for residual $\Phi_{l}^{E}$ (red solid line) and the corresponding Gaussian distribution (blue dashed line). Here $\mu_{1}$ is the mean value of $\Phi_{l}^{E}, \sigma_{1}$ is the standard deviation of $\Phi_{l}^{E}, \mu_{2}$ is the mean value of the residual of $\Phi_{l}^{E}$, and $\sigma_{2}$ is the standard deviation of the residual of $\Phi_{l}^{E}$.

In Fig. 8, we show ensemble-averaged $\Phi_{l}^{H}$ conditioned on local velocity divergence and local helicity with a different filter width. The ensemble-averaged $\Phi_{l}^{H}$ changes monotonously conditioned on both local velocity divergence and local helicity. Similar to the result in Fig. 6(a), the compression regions play a sink role for total helicity represented by positive $\Phi_{l}^{H}$. In Fig. 8(b), we see that when helicity is negative, the ensemble-averaged $\Phi_{l}^{H}$ is also negative and vice versa. Hence we conclude that $\Phi_{l}^{H}$ plays a source role for positive and negative helicity, respectively, which is opposite to the sink role of $\Phi_{l}^{E}$ for kinetic energy.

\section{THE ROLE OF A SHOCKLET}

Another question arises when faced with shocklets. In compressible turbulence, especially with high $\mathrm{M}_{t}$, the inevitable issue is a shocklet, which is unique in compressible turbulence. The appearance of a shocklet gives rise to many challenging problems that cannot be fully explained. As for the role of pressure terms in the turbulence cascade mentioned above, we did not analyze the effect of a shocklet. So what is the fundamental physical mechanism of a shocklet? In this section, we try to answer that question.

There are a large number of compression regions and rarefaction regions in flows, and these cancellations among them lead to approximately negligible contributions to filtered results for $\Phi_{l}^{E}$ [13]. In Fig. 9, we show the results of a two-dimensional slice of $1024^{2}$ and the normalized PDF of $\Phi_{l}^{E}$, residual $\left(\Phi^{E}-\Phi_{l}^{E}\right)$, and the corresponding Gaussian distribution. In contrast to $\Phi_{l}^{E}$, the residual is one order higher at pointwise value while it is one order lower at mean value. This regularity confirms the above opinion about cancellations among compression regions and rarefaction regions. In addition, the distribution of the residual is far from the corresponding Gaussian distribution, especially at the left tail, which proves that the compression region is dominant in our numerical simulations.

Likewise, we analyze a two-dimensional slice and the PDF of $\Phi_{l}^{H}$ in Fig. 10. We could get the same pattern as in Fig. 9. The residual is one order higher pointwise but one 

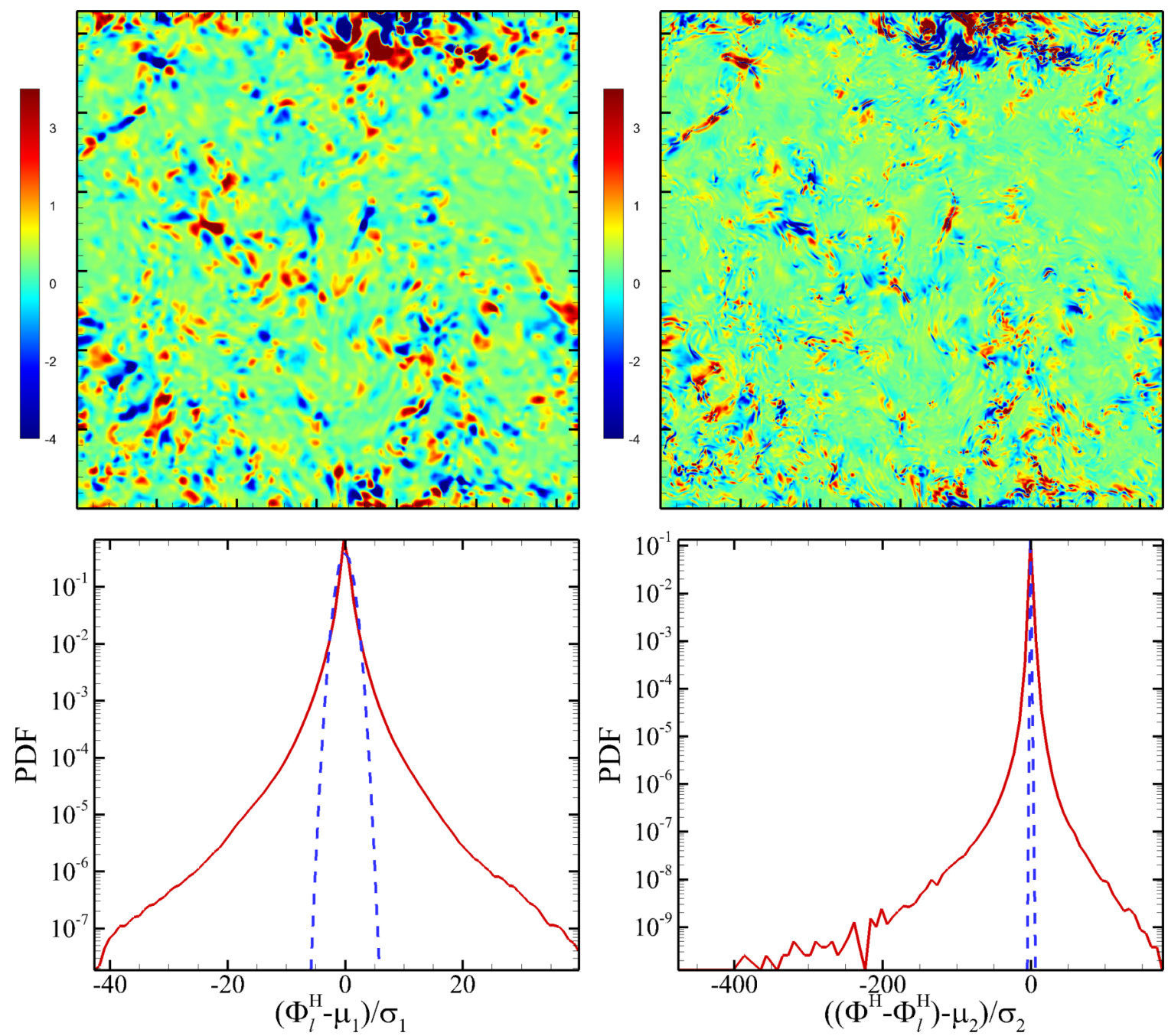

FIG. 10. Top left: two-dimensional slice of pointwise $\Phi_{l}^{H}$. Bottom left: normalized distribution curve for $\Phi_{l}^{H}$ (red solid line) and the corresponding Gaussian distribution (blue dashed line). Top right: two-dimensional slice of residual pointwise $\Phi_{l}^{H}$. Bottom right: normalized distribution curve for residual $\Phi_{l}^{H}$ (red solid line) and the corresponding Gaussian distribution (blue dashed line). Here $\mu_{1}$ is the mean value of $\Phi_{l}^{H}, \sigma_{1}$ is the standard deviation of $\Phi_{l}^{H}, \mu_{2}$ is the mean value of the residual of $\Phi_{l}^{H}$, and $\sigma_{2}$ is the standard deviation of the residual of $\Phi_{l}^{H}$.

order lower at mean value, showing the cancellations among compression and rarefaction regions. This "coarse-graining" method excludes small scales successfully and preserves the features of large scales. Comparing the results in Figs. 9 and 10 , we find that the distribution of residual $\Phi_{l}^{H}$ deviates more from Gaussian distribution. This phenomenon confirms the previous opinion that the fluctuating amplitude of $\Phi_{l}^{H}$ is larger than that of $\Phi_{l}^{E}$. The gradient of the inverse of density only shines through small scales, and that is the role of the shocklet for $\Phi_{l}^{H}$. Hence we can extend the role of skocklets for $\Phi_{l}^{E}$ to $\Phi_{l}^{H}$ successfully.

\section{CONCLUSIONS AND DISCUSSIONS}

The present study was designed to determine the role of $\Phi_{l}^{E}$ and $\Phi_{l}^{H}$ for the joint cascade of kinetic energy and helicity in compressible helical turbulence. The method of "coarse-graining" provides us with a deeper insight into the fundamental physical mechanism of compressible turbulence.
This study has been one of the first attempts to thoroughly examine the effect of $\Phi_{l}^{H}$ extended from $\Phi_{l}^{E}$.

We found that $\Phi_{l}^{H}$ operates only in large scales statistically similar to $\Phi_{l}^{E}$, but $\Phi_{l}^{H}$ contributes a smaller cumulative effect and its effective range is smaller than $\Phi_{l}^{E}$. From the perspective of their PDF, the PDF of $\Phi_{l}^{H}$ does not show an obvious skewness to positive or negative values, and both tails decrease with an increase of the filter width. These results imply that there exists a longer inertial subrange for the helicity cascade, in contrast to the kinetic energy cascade. Even though the pressure term is very sensitive to the appearance of a shocklet, the cancellations between the compression and rarefaction regions make a negligible contribution to $\Phi_{l}^{H}$. Hence, the presence of a shocklet could not affect our conclusions.

The effects of helicity on both $\Phi_{l}^{E}$ and $\Phi_{l}^{H}$ are investigated in detail. High enough helicity hinders the energy conversion process of kinetic energy transferring to internal energy and promotes the transfer of internal energy to kinetic energy through the effect of the pressure term. This discovery may 
provide a method of improving the energy efficiency for engines, gas turbines, and so on. Similar to the contribution of $\Phi_{l}^{E}$ for kinetic energy, $\Phi_{l}^{H}$ also dissipates the total helicity on the whole. But $\Phi_{l}^{H}$ plays a source role both for positive and negative helicity, respectively.

This new understanding should help to improve predictions of accuracy for compressible turbulence modeling in LES. The larger inertial subrange of the helicity cascade corresponds to a wider range of application of turbulence modeling in LES based on the helicity cascade. The above investigations of pressure terms eliminate obstacles when extending some theories of the helicity cascade in incompressible turbulence to compressible turbulence, which may pave the way for further studies focusing on the properties of helicity in compressible turbulence.

\section{ACKNOWLEDGMENTS}

This work was supported by the National Natural Science Foundation of China (NSFC Grants No. 91852203 and No. 11472278), the National Key Research and Development Program of China (2016YFA0401200), and Science Challenge Project (TZ2016001). The authors thank National Supercomputer Center in Tianjin (NSCC-TJ), and National Supercomputer Center in GuangZhou (NSCC-GZ) for providing computer time.
[1] R. Samtaney, D. I. Pullin, and B. Kosović, Phys. Fluids 13, 1415 (2001).

[2] U. Frisch, Turbulence: The Legacy of A. N. Kolmogorov (Cambridge University Press, Cambridge, England, 1995).

[3] J. W. Armstrong, B. J. Rickett, and S. R. Spangler, Astrophys. J. 443, 209 (1995).

[4] H. Xu, H. Li, D. C. Collins, S. Li, and M. L. Norman, Astrophys. J. 698, L14 (2009).

[5] J. Wang, Y. Shi, L.-P. Wang, Z. Xiao, X. T. He, and S. Chen, J. Fluid Mech. 713, 588 (2012).

[6] J. Wang, Y. Yang, Y. Shi, Z. Xiao, X. T. He, and S. Chen, Phys. Rev. Lett. 110, 214505 (2013).

[7] J. Wang, T. Gotoh, and T. Watanabe, Phys. Rev. Fluids 2, 023401 (2017).

[8] J. Wang, T. Gotoh, and T. Watanabe, Phys. Rev. Fluids 2, 053401 (2017).

[9] J. Wang, M. Wan, S. Chen, C. Xie, and S. Chen, Phys. Rev. E 97, 043108 (2018).

[10] J. Wang, M. Wan, S. Chen, and S. Chen, J. Fluid Mech. 841, 581 (2018).

[11] D. Zhao and H. Aluie, Phys. Rev. Fluids 3, 054603 (2018).

[12] H. Aluie, Phys. Rev. Lett. 106, 174502 (2011).

[13] H. Aluie, S. Li, and H. Li, Astrophys. J. 751, L29 (2012).

[14] H. Aluie, Physica D 247, 54 (2013).

[15] A. Brissaud, Phys. Fluids 16, 1366 (1973).

[16] H. K. Moffatt and A. Tsinober, Annu. Rev. Fluid Mech. 24, 281 (1992).

[17] T. Gomez, H. Politano, and A. Pouquet, Phys. Rev. E 61, 5321 (2000).

[18] J. C. Andre and M. Lesieur, J. Fluid Mech. 81, 187 (1977).

[19] V. Borue and S. A. Orszag, Phys. Rev. E 55, 7005 (1997).

[20] Q. Chen, S. Chen, and G. L. Eyink, Phys. Fluids 15, 361 (2003).

[21] Q. Chen, S. Chen, G. L. Eyink, and D. D. Holm, Phys. Rev. Lett. 90, 214503 (2003).

[22] L. Biferale, S. Musacchio, and F. Toschi, Phys. Rev. Lett. 108, 164501 (2012).

[23] L. Biferale, S. Musacchio, and F. Toschi, J. Fluid Mech. 730, 309 (2013).

[24] G. Sahoo, A. Alexakis, and L. Biferale, Phys. Rev. Lett. 118, 164501 (2017).

[25] R. Stepanov, E. Golbraikh, P. Frick, and A. Shestakov, Phys. Rev. Lett. 115, 234501 (2015).
[26] H. K. Moffatt, J. Fluid Mech. 35, 117 (1969).

[27] H. K. Moffatt, Proc. Natl. Acad. Sci. (USA) 111, 3663 (2014).

[28] M. Kessar, F. Plunian, R. Stepanov, and G. Balarac, Phys. Rev. E 92, 031004 (2015).

[29] M. Lautenschlager, D. P. Eppel, and W. C. Thacker, Beitr. Phys. Atmosph. 61, 87 (1988).

[30] D. K. Lilly, J. Atmos. Sci. 43, 126 (1986).

[31] T. Gotoh and T. Nakano, J. Stat. Phys. 113, 855 (2003).

[32] R. K. Bikkani and S. S. Girimaji, Phys. Rev. E 75, 036307 (2007).

[33] A. Pumir, H. Xu, G. Boffetta, G. Falkovich, and E. Bodenschatz, Phys. Rev. X 4, 041006 (2014).

[34] M. Wilczek and C. Meneveau, J. Fluid Mech. 756, 191 (2014).

[35] J. M. Lawson and J. R. Dawson, J. Fluid Mech. 780, 60 (2015).

[36] P. Bechlars and R. D. Sandberg, in Progress in Turbulence VII, 1st ed., edited by R. Örlü, T. Alessandro, O. Martin, and P. Joachim, Springer Proceedings in Physics (Springer, New York, 2016), Vol. 196, Chap. 3, pp. 17-22.

[37] M. Danish, S. Suman, and B. Srinivasan, Phys. Fluids 26, 126103 (2014).

[38] J. Wang, L.-P. Wang, Z. Xiao, Y. Shi, and S. Chen, J. Comput. Phys. 229, 5257 (2010).

[39] W. Sutherland, Philos. Mag. 36, 507 (1992).

[40] D. S. Balsara and C. W. Shu, J. Comput. Phys. 160, 405 (2000).

[41] S. K. Lele, J. Comput. Phys. 103, 16 (1992).

[42] H. Miura and S. Kida, Phys. Fluids 7, 1732 (1995).

[43] F. Waleffe, Phys. Fluids A 4, 350 (1992).

[44] M. V. Melander and F. Hussain, Phys. Fluids A 5, 1992 (1993).

[45] G. Sahoo, M. D. Pietro, and L. Biferale, Phys. Rev. Fluids 2, 024601 (2017).

[46] J. Bec and K. Khanin, Phys. Rep. 447, 1 (2007).

[47] S. Lee, S. K. Lele, and P. Moin, Phys. Fluids A 3, 657 (1991).

[48] A. Favre, C. R. Acad. Sci. Paris 246, 2573 (1958).

[49] G. L. Eyink, Physica D 207, 91 (2005).

[50] P. D. Ditlevsen and P. Giuliani, Phys. Rev. E 63, 036304 (2001).

[51] P. D. Ditlevsen and P. Giuliani, Phys. Fluids 13, 3508 (2001). 